\title{
A study of the influence of REV variability in double-scale FEM $\times$ DEM analysis
}

\author{
Ghassan Shahin $^{1,2}$, Jacques Desrues ${ }^{2,1, *, \dagger}$, Stefano Dal Pont ${ }^{1,2}$, Gaël Combe ${ }^{1,2}$ \\ and Albert Argilaga ${ }^{1,2}$ \\ ${ }^{1}$ Université Grenoble Alpes, 3SR, F-38000 Grenoble, France \\ ${ }^{2}$ CNRS, 3SR, F-38000 Grenoble, France
}

\begin{abstract}
SUMMARY
In this work, the consequences of using several different discrete element granular assemblies for the representation of the microscale structure, in the framework of multiscale modeling, have been investigated. The adopted modeling approach couples, through computational homogenization, a macroscale continuum with microscale discrete simulations. Several granular assemblies were used depending on the location in the macroscale finite element mesh. The different assemblies were prepared independently as being representative of the same material, but their geometrical differences imply slight differences in their response to mechanical loading. The role played by the micro-assemblies, with weaker macroscopic mechanical properties, on the initiation of strain localization in biaxial compression tests is demonstrated and illustrated by numerical modeling of different macroscale configurations. Copyright (C) 2016 John Wiley \& Sons, Ltd.
\end{abstract}

Received 5 August 2015; Revised 15 December 2015; Accepted 23 December 2015

KEY WORDS: multiscale modeling; FEM $\times$ DEM; representative elementary volume (REV); damage analysis; strain localization; granular material

\section{INTRODUCTION}

Multiscale modeling of granular materials has received an increasing attention in the recent years [1-6] as these approaches allow to naturally embed a refined description of the complexity of the material into a full structural engineering problem. Moreover, these techniques allow to efficiently overcome the common drawbacks of conventional modeling strategies [7, 8]. In this regard, finite elements-based approaches allow to treat large-scale problems; however, the application of the method to complex materials (such as geomaterials) may be unsatisfactory because of the difficulties arising in the definition of an adequate mechanical constitutive law. On the other hand, the discrete element method (DEM) [9-11] is usually considered particularly effective to capture the nature of geomaterials and properly describe their constitutive behavior. However, the need for a large number of particles to simulate a full-engineering problem imposes some restrictions to the application of this method [12].

Recently, the two methods have been coupled in the framework of a multiscale numerical homogenization approach to exploit the efficiency of FEM at solving boundary value problems at structural level and the capability of DEM to capture complex material behaviors. Such an approach is usually known as FEM $\times$ DEM method. Several multiscale modeling schemes have been proposed. Kaneko et al. $[1,13]$ employed the mathematical homogenization theory for the construction of a multiscale modeling approach. Miehe et al. [3, 14] proposed an original scheme for quasi-static homogenization of granular microstructures and its embedding into a two-scale modeling framework.

\footnotetext{
*Correspondence to: Jacques Desrues, Laboratoire 3SR, Domaine Universitaire, BP53, 38041 Grenoble Cedex 9, France.

${ }^{\dagger}$ E-mail: jacques.desrues@3sr-grenoble.fr 
Meier et al. $[2,15]$ built an FEM $\times$ DEM scheme employing the continuum-based Taylor assumption at the microscale level. In these methods, the DEM is used to derive an equivalent mechanical law for the microstructure behavior. Andrade et al. [5] and Avial et al. [16] developed a discretecontinuum approach based on a numerical homogenization scheme in which friction and dilatation at the microstructure are transferred to the elasto-plasticity continuum at the macroscale level. Nitka et al. [4, 17], Nguyen et al. [18, 19], Guo and Zhao [6, 20], and Desrues et al. [21] applied the concept of the representative elementary volume (REV) to build a fully coupled multiscale FEM $\times$ DEM approach based on computational homogenization. The stress state at the macroscale level is obtained, for each Gauss point, from the associated DEM granular assembly (REV).

The efficiency of the method has been explored through several study cases, basically consisting of monotonic compression biaxial test $[1,3,5,16,19,20,22]$, cyclic simple shear test [20], hollow tube test [21], and slope stability test [15], in addition to the retaining wall problem and the classical footing problem [23]. These numerical experiments were run using both cohesive and disperse materials and considering only a single DEM granular assembly to represent the material microstructure constitutive behavior over the whole specimen. However, computerized tomography scans (e.g., Figure 1) reveal a strong randomness in the distribution of the particles over a sample volume, thus questioning the assumption of a single REV. According to experimental observation, the use of a single microstructure volume periodically distributed over the entire specimen may lead to the loss of some distinct features of the considered geomaterial.

The scope of the paper is to move towards a more realistic representation of geomaterials by considering, in the framework of a FEM $\times$ DEM approach, different REVs at the microstructural level. The used REVs are representative of the same material, (i.e., the same material properties with random realization of the same grain size distribution), but they differ with respect to their geometry (i.e., the positioning of the particles is different), as suggested by the experimental evidence.

The paper is structured as follows. Section 2 briefly summarizes the adopted multiscale FEM $\times$ DEM modeling approach. Section 3 presents the preparation algorithm of the granular assemblies and provides identification and selection criteria of the granular assemblies. Section 4 describes the FEM $\times$ DEM modeling details and the biaxial test procedures. Section 5 shows pure DEM and homogeneous FEM $\times$ DEM simulations. Section 6 proceeds towards the focal issue in this work to carry out a set of inhomogeneous FEM $\times$ DEM simulations. Section 7 uses the DEM REV of the weak mechanical properties to introduce material imperfection into homogeneous simulation. The work is then concluded, and some perspectives are presented.

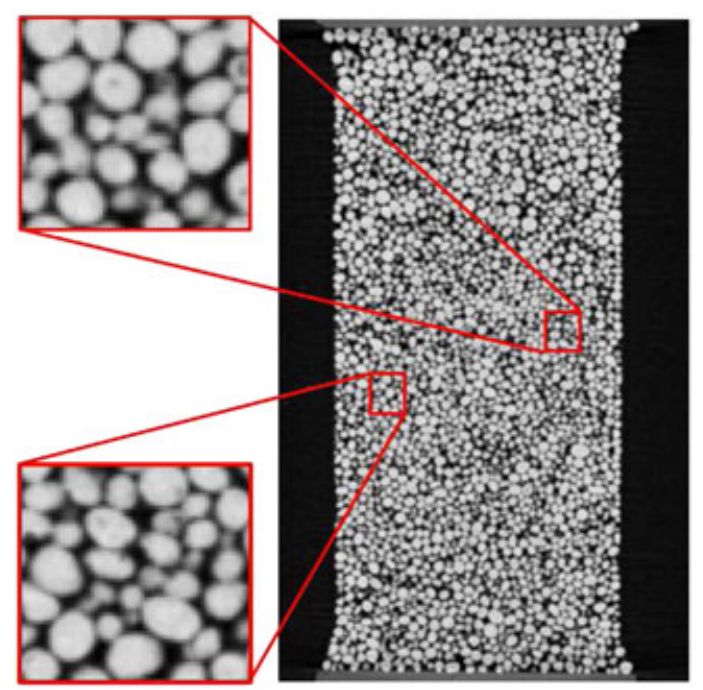

Figure 1. A computed tomography scan of dry sand laboratory sample [24]. Although it was prepared using the same material, this homogeneous sample implies strong fluctuations in microstructures geometry. 


\section{MULTISCALE FEM $\times$ DEM MODELING APPROACH}

In this work, the multiscale FEM $\times$ DEM modeling approach proposed by Nitka et al. [4] and then improved in Nguyen et al. [18, 19] and Desrues et al. [21] has been adopted as a general framework. In this method, a quasi-static finite strain macroscopic continuum is coupled with discrete microstructures defining the constitutive behavior of the material. The macrostructure boundary value problem is described by means of FEM, whereas the local constitutive relation at the Gauss point level is determined by a DEM granular assembly that is considered as an REV. The local REV is representative of the material behavior, and each REV has its own stress-strain evolution (like a standard Gauss point in FEM). In the sequel, the modeling approach is restricted to the 2D simulation at both macroscopic and microscopic levels.

The constitutive law at the macrostructural level gives the stress state $\sigma_{i j}(t)$ as a function of the displacement gradient history $h_{k l}$ :

$$
\sigma_{i j}(t)=\Gamma^{t}\left\{h_{k l}(\tau), \tau \in(0, t)\right\}
$$

The DEM-FEM coupling is obtained through a computational homogenization method following the typical step shown in Figure 2. As in standard finite elements, the displacement gradient increment $\delta h_{k l}$ is applied at the Gauss point level to obtain the corresponding stress state. In the FEM $\times$ DEM scheme, the displacement gradient increment acts as an updated boundary condition on the discrete element REV at the Gauss point level. The subsequent discrete element numerical simulation acts as a material constitutive relation by returning the new stress state at the FE-macro level. The updated Cauchy stress state is obtained through the standard Weber [25] homogenization formulation:

$$
\sigma_{i j}=\frac{1}{S} \cdot \sum_{(p, q) \in c} f_{i}^{q / p} \otimes l_{j}^{p / q}
$$

where $S$ is the area of the assembly, $f^{q / p}$ and $l^{p / q}$ are, respectively, the interparticle forces and the branch links of the $p, q$ grains. $c$ is the set containing all the contacts in the granular assembly.

Computational homogenization techniques typically rely on a scales-separation assumption $[8,26,27]$, thus requiring that the characteristic lengths of the spatial variation of macroscopic fields are much larger than the characteristic size of the embedded microstructure. This assumption holds as long as localization does not occur. As soon as a localization band develops, the microstructural element is no longer statistically representative (strictly speaking, a generalized Microstructural Element Volume rather than an RVE should be introduced). The interested reader should refer to, for example, Reference [26] or, more recently, Reference [27].

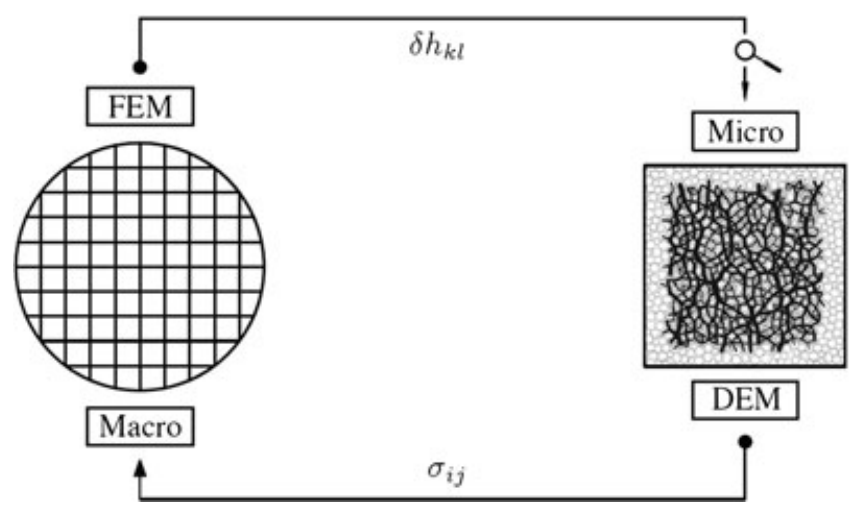

Figure 2. Computational homogenisation scheme [18]. FEM, Finite element method. 
Table I. The equilibrium criteria and the six levels of equilibrium conditions of DEM computation.

\begin{tabular}{lcccccc}
\hline Level of equilibrium & 0 & 1 & 2 & 3 & 4 & 5 \\
\hline Min number of iterations & 10000 & 10000 & 10000 & 10000 & 10000 & 10000 \\
Max kinetic energy & $1.0 \mathrm{E}-2$ & $1.0 \mathrm{E}-5$ & $1.0 \mathrm{E}-8$ & $1.0 \mathrm{E}-8$ & $1.0 \mathrm{E}-8$ & $1.0 \mathrm{E}-8$ \\
Max proportion of sliding contacts & $10 \%$ & $10 \%$ & $10 \%$ & $10 \%$ & $1 \%$ & $0 \%$ \\
Max proportion of grains with single contact & $10 \%$ & $10 \%$ & $10 \%$ & $10 \%$ & $1 \%$ & $0 \%$ \\
Max resultant/minimal forces acting on each grain & $1.0 \mathrm{E}-2$ & $1.0 \mathrm{E}-2$ & $1.0 \mathrm{E}-2$ & $1.0 \mathrm{E}-2$ & $1.0 \mathrm{E}-2$ & $1.0 \mathrm{E}-6$ \\
\hline
\end{tabular}

The used FEM code [28] is a finite strain implementation of the standard finite element method [29], and the DEM code is an in-house implementation of the standard discrete element method [9]. The DEM model assumes that all grains interact under linear-elastic normal and tangential stiffness. Coulomb-type friction is introduced into contact system, and energy is dissipated through a viscous damping mechanism.

\subsection{FEM algorithm}

The FEM solution is integrated by an adaptive step-by-step strategy in which the solution of a typical step is obtained by means of Newton-Raphson method [30]. The Newton-Raphson method requires the determination of the consistent tangent operator (CTO) $C_{i j k l}$ for each step in order to integrate the solution.

$$
C_{i j k l}=\frac{d \sigma_{i j}}{d h_{k l}}
$$

The CTO is numerically computed for the first quasi-elastic steps using the perturbation method [19]. When the material behavior becomes strongly nonlinear (at about $0.5 \%$ axial strain), the CTO computation becomes numerically less efficient. Therefore, a different strategy is adopted, and the tangent operator is considered as the average of the tangent operators of the first 10 steps. As an auxiliary operator is used, the quadratic convergence, which is supposed to be obtained with a Newton-Raphson method, is lost, and a larger number of iterations will be required. Such a modified Newton-Raphson approach, however, proved to be more robust than the full Newton's method [18] based on the computations of the CTO. This iterative scheme is associated with two different convergence criteria based on out-of-balance forces and displacement decrement (Appendix A).

\subsection{DEM algorithm}

The displacement gradient increment $\delta h_{k l}$ is transferred from the FE macroscale and applied to the DE microstructure through an explicit step-by-step integration scheme. The application of $\delta h_{k l}$ is conducted with fixed time step $\delta t$ that is computed based on the dynamic properties of the contacted particles. After the application of the strain, the assembly is submitted to a relaxation phase that ends once the assembly reaches an equilibrium state. The equilibrium of the assembly is estimated through the five criteria that are illustrated in Table I. At the granular level, the solution of motion equation is obtained by means of a predictor-corrector [31] integration method of order 3 .

\section{DEM REVS SIMULATIONS}

In the framework of the FEM $\times$ DEM modeling approach, the DEM granular assembly is used to define the constitutive response of the considered material. Preparation procedures of the DEM assembly, to be used as REV in this process, are of great importance because of their strong effects 
on the mechanical properties of the final assembly [10, 11]. Hereafter, a detailed description of the followed preparation procedures is presented, and a set of granular assemblies is generated accordingly. A subset of granular assemblies is then selected, and a biaxial test simulation is proposed, in Section 5.1, to explore their mechanical behavior.

\subsection{The DEM model}

A standard DEM model $[10,11]$ has been used in this work. The model assumes that all rigid circular grains interact when they are in contact. The normal contact force is given as $f_{n}=-K_{n} \delta$, where $K_{n}$ is the normal contact stiffness and $\delta$ is overlapping magnitude ( $\delta<0$ when the contact occurs). The tangential force increment is given as $\delta f_{t}=K_{t} . \delta U_{t}$, where $K_{t}$ is the tangential stiffness and $\delta U_{t}$ is an increment of tangential relative displacement computed at the contact point. The total tangential force, $f_{t}$, is the sums over the time step $\delta t$. The Coulomb friction coefficient is used to limit $f_{t}$ such that $\left|f_{t}\right| \leqslant \mu f_{n}$, where $\mu$ is the contact angle of friction. The kinetic energy of the system is dissipated through viscous dampers introduced to each contact system. If $m_{i}$ and $m_{j}$ are the mass of the two particles in contact, damping coefficient is defined as a proportion of the critical damping, $\eta$, which is computed using the relation $\eta=2 \times\left(K_{n} \times \frac{m_{i} \times m_{j}}{m_{i}+m_{j}}\right)^{0.5}$.

\subsection{DEM REV generation}

The assembly is formed of 400 particles, consistently with the guidelines provided by Nguyen et al. $[18,19]$ and Guo and Zhao [6, 20]. This choice constitutes a good compromise between the computational cost and the problem stability. In order to obtain homogeneous assemblies, the preparation procedures were performed in the absence of gravity, and periodic boundary conditions were retained in the horizontal and vertical directions [10, 11]. In this work, the simulation is focused on a dense frictional granular material (without cohesion). To obtain a dense assembly, the inter-particle friction coefficient has been set to zero during the preparation step as the particles can slide and fill the volume as much as possible. In addition, the grain size range has been taken as $R_{\max } / R_{\min }=2.5$.

Discrete element method granular assemblies have been generated following the algorithm proposed in [10] and [11]. Starting from a given particle size range and a given number of particles, a random generator places the particles on a regular grid of unit length modulus, (Figure 3(a)). The particles are then submitted to a random velocity field. The particles move and interact as rigid bodies within a fixed size container. No energy dissipation is introduced during this process. When particles have been shaken enough (i.e., each particle is displaced cumulatively 100 times its

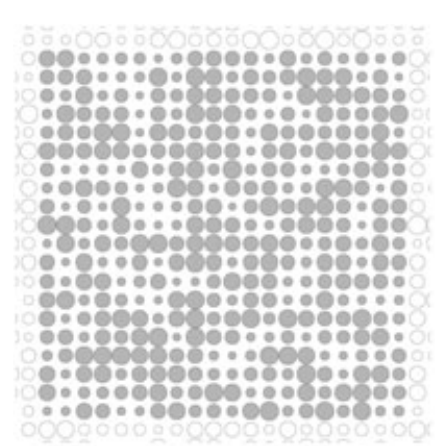

(a)

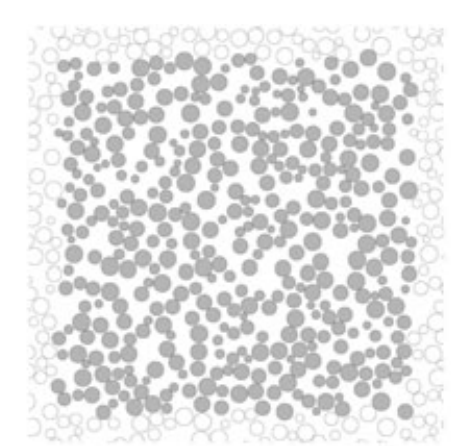

(b)

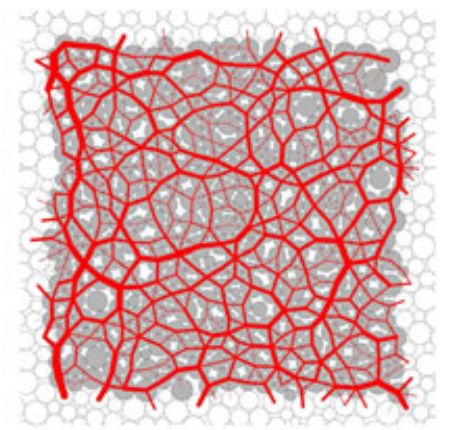

(c)

Figure 3. The three stages of the granular assembly preparation with periodic boundary conditions. (a) The ordered configuration of the assembly; (b) the granular assembly after shaking; and (c) the final configuration after the isotropic compaction of the granular assembly. 
Table II. The given values of DEM mechanical parameters.

\begin{tabular}{lc}
\hline Parameter & Value \\
\hline Normal stiffness coefficient $\kappa=K_{n} / P$ & 1000 \\
Tangent/normal stiffness coefficients $K_{t} / K_{n}$ & 1 \\
Coulomb friction & 0.5 \\
Viscous damping coefficient & 0.95 \\
\hline$\kappa$ is the normal stiffness coefficient normalized by confinement \\
pressure P. Damping coefficient is a proportion of, $\eta$, the critical \\
damping of contact system.
\end{tabular}

diameter), their velocity is set to zero, (Figure 3(b)). The resultant granular packing, which has a gas-like configuration, is then subjected to a strain-controlled isotropic compression phase leading to the desired granular assembly, (Figure 3(c)).

Following these procedures, more than 20 granular assemblies have been generated using the material mechanical properties presented in Table II. The proposed procedure allows to generate REVs characterized by major differences in particles positioning but only minor fluctuations in particle size distribution. The generated REVs can therefore be considered as representative of the same material. Because the objective of this study is to investigate the consequences of using different DEM REVs, each of these granular assemblies will be given an identity based on the criteria that are presented in the following section.

\subsection{Granular assembly identity and selection criteria}

Radjai and Dubois [10] introduced a number of criteria to determine the identity of a given granular assembly. They defined a set of internal variables that distinguish one granular assembly from another. Among these variables, packing fraction $(\mathrm{PF})$ and coordination number $(\mathrm{CN})$ have been retained hereafter. The PF describes the proportion of solids volume to the overall assembly volume, whereas the $\mathrm{CN}$ represents the average number of inter-particles contacts per particle in the packing. If $N_{c}$ is the total number of contacts, and $n$ is the number of particles in contact, then $C N=2 N_{c} / n$.

The generated assemblies exhibit a Gaussian distribution of PF and $\mathrm{CN}$ that can be formulated as $P F=0.8157 \pm 0.004$ and $C N=4.153 \pm 0.015$. The small deviation of $\mathrm{PF}$ and $\mathrm{CN}$ proves the efficiency of the proposed procedure in generating granular assemblies representative of the same material. Such a small deviation, however, excludes the typical correlation with the strength peak, as the relation between strength peak and these two variables exhibits a random scattering within a small window of variation. This scattering can significantly be affected by the number of grains; a smaller number of grains promotes the role of geometry, as any small variation in the fabric would result in significant changes in the strength peak. Therefore, the introduced randomness in geometry factors at preparation process will show, in the next section, a clear influence on the strength peak of the granular assemblies.

As aforementioned, the idea of using different granular assemblies is to represent the random geometrical variations of the microstructure in soil sample, apart from any deviation in the mechanical properties. Thus, the selection of the granular assemblies has to be conducted independently from their mechanical properties. Given that the mechanical properties (strength peak) of the assemblies exhibit a random scattering within a small variation of the internal variables, considering $\mathrm{PF}$ and $\mathrm{CN}$ as selection criteria can offer such sort of independent process. The assemblies corresponding to the mean value \pm a standard deviation of $\mathrm{PF}$ and $\mathrm{CN}$ have been selected. The assemblies corresponding to the mean value of PF plus/minus a standard deviation are denoted by PF-A/PF-B, respectively. Whereas, the assemblies corresponding to the mean value of $\mathrm{CN}$ plus/minus a standard deviation are denoted $\mathrm{CN}-\mathrm{A} / \mathrm{CN}-\mathrm{B}$, respectively.

Another significant variable has to be considered. In the procedures, compaction process is conducted as the normal strain components are imposed, but not the shear strain component. Instead, 
zero shear strain is imposed (i.e., orthogonal wall driving). Even though, at the end of the preparation stage, the obtained assemblies always show a slight deviation from isotropy. Equation (4) shows a comparison between the target stress state (left matrix) and the homogenized internal stress state using Equation (2) (right matrix) for an arbitrarily chosen packing from the generated assemblies. This deviation from isotropy can be attributed to the finite number of grains in the granular assemblies. Despite its small magnitude, this deviatoric stress component will affect the mechanical behavior once introduced in the double-scale computations of FEM $\times$ DEM scheme, as it introduces an initial bias to simulation specimen [20]. In the following, this deviatoric stress component will be termed as remaining shear stress (RS). To investigate the effects of RS on the interplay between the microstructure and macrostructure, it has been considered as a third selection criterion. The assemblies corresponding to both the maximum positive and the minimum positive RS have been selected and denoted by RS-A and RS-B, respectively. In fact, the assembly associated to the minimum RS has already been considered for the $\mathrm{CN}$ criterion. Replicating the use of the same assembly might render some difficulties at reading the plots. Therefore, another one has been chosen, namely, an assembly with RS magnitude close to zero. Table III shows the chosen assemblies and their notation, in addition to the associated values of PF, CN, and RS.

$$
\left(\begin{array}{ll}
1 & 0 \\
0 & 1
\end{array}\right) \neq\left(\begin{array}{cc}
1 & +0.07591 \\
+0.07591 & 1
\end{array}\right)
$$

Table III. The selected DEM REVs based on the three criteria, PF, CN, and RS, for microstructure representation in multiscale simulation.

\begin{tabular}{llcccc}
\hline \multirow{2}{*}{ Notation } & \multirow{2}{*}{ Meaning } & \multirow{2}{*}{ Target Value } & \multicolumn{3}{c}{ Actual values } \\
\cline { 4 - 6 } & & & PF & CN & RS \\
\hline PF-A & Mean+STD & 0.8193 & $\mathbf{0 . 8 1 9 4}$ & 4.1474 & 0.0445 \\
PF-B & Mean-STD & 0.8122 & $\mathbf{0 . 8 1 2 9}$ & 4.1575 & -0.0206 \\
CN-A & Mean+STD & 4.1685 & 0.8150 & $\mathbf{4 . 1 7 0 1}$ & -0.0081 \\
CN-B & Mean-STD & 4.1381 & 0.8147 & $\mathbf{4 . 1 3 7 6}$ & 0.0015 \\
RS-A & Maximum & 0.0759 & 0.8125 & 4.1705 & $\mathbf{0 . 0 7 5 9}$ \\
RS-B & Minimum & $\mathbf{0 . 0 0 1 5}$ & $\mathbf{0 . 8 1 7 0}$ & 4.1399 & $\mathbf{0 . 0 0 7 9}$ \\
\hline
\end{tabular}

$\mathrm{PF}$, packing fraction; $\mathrm{CN}$, coordination number; RS, remaining shear stress.

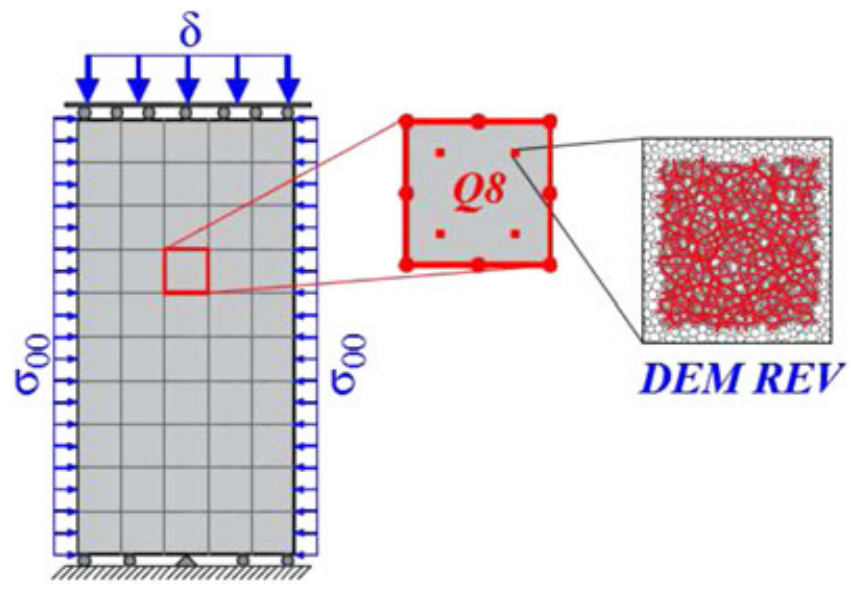

Figure 4. The finite element model and its boundary conditions used at the macroscale level of the multiscale simulation specimens of aspect ratio 2 discretized using $(5 \times 10) 50 \mathrm{Q} 8$ finite elements. 
Table IV. The adopted computational parameters values based on the performed sensitivity study.

\begin{tabular}{cccc}
\hline IN & LE & PM & ED|EF \\
\hline $1.0 \mathrm{E}-4$ & 2 & $15.0 \mathrm{E}-6$ & $0.01 \mid \mathrm{NA}$ \\
\hline
\end{tabular}

IN, inertial number; LE, level of equilibrium; PM, perturbation magnitude; ED, displacement-based convergence criterion; $\mathrm{EF}$, force-based convergence criterion.

\section{FEM $\times$ DEM MODELING SETUP}

The biaxial test is the equivalent for 2D modeling of the so-called triaxial test widely used for the characterization of granular media behavior $[32,33]$. Herein, the idea is to use the FEM $\times$ DEM approach to develop a biaxial test simulation. A detailed description of the FEM $\times$ DEM model and the applied biaxial test procedures are presented hereafter.

\subsection{The numerical specimen}

The macroscale of the specimen to be modeled is discretized into $5 \times 10$ finite elements mesh (aspect ratio 2). The eight-nodes quadrilateral element with four integration Gauss points (Q8) is retained in the study. The finite element mesh and its boundary conditions are illustrated in Figure 4. Each Gauss point in this mesh is associated with a specific DEM assembly. The six DEM assemblies already presented in Table III will be used for this purpose.

\subsection{Numerical experiment setup}

The FEM $\times$ DEM numerical study is focused on a standard monotonic compressive biaxial test. In its initial state, the specimen is subjected to an isotropic compression. Thereafter, the horizontal confinement pressure is held constant and the deviatoric stress is imposed through strain-controlled vertical compressive loading, for more details see, for example, Desrues and Viggiani [32].

The numerical procedures of both FEM and DEM, presented in Section 2, imply the choice of a set of computational parameters, which play a major role on the efficiency of the analysis: the convergence rate at both macroscale and microscale. These parameters are the inertial number (IN) and the level of equilibrium (LE) conditions for the DEM scheme, the perturbation magnitude, and the precision level $\left(E_{F}\right.$ and $E_{D}$ ) for the FEM scheme. For more details about these four parameters, see Appendix A. These parameters were identified via a sensitivity analysis that is presented in Appendix A and summarized in Table IV.

\section{HOMOGENEOUS MULTISCALE SIMULATION}

In this part, several FEM $\times$ DEM biaxial test simulations are performed using the REVs introduced in Table III. A unique DEM REV is used in each simulation, thus obtaining an initially homogeneous specimen having the same mechanical properties over the entire domain. Before conducting the FEM $\times$ DEM simulations, the behavior of the used DEM REVs is investigated by means of pure DEM simulation of biaxial test. These simulations describe the behavior of the material itself, and they will be used for comparison with the multiscale FEM $\times$ DEM simulations that exhibit, after the localization of strain field, structural behavior rather than material behavior.

\subsection{Material behavior - pure DEM simulations}

The behavior of the material used in our numerical experiments is first explored through a pure DEM simulation. Each DEM assembly is subjected to a biaxial loading path up to $8 \%$ axial strain. Figure 5 shows the global response of the six assemblies plotted as a function of the deviatoric stress $q$ normalized by the confinement pressure $\sigma_{00}$ versus the applied axial strain. The deviatoric 


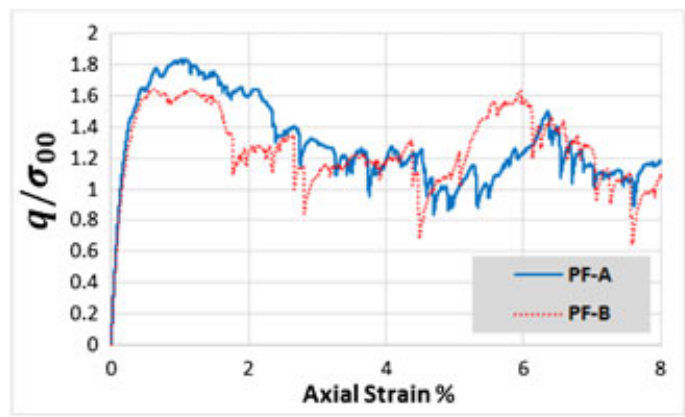

(a)

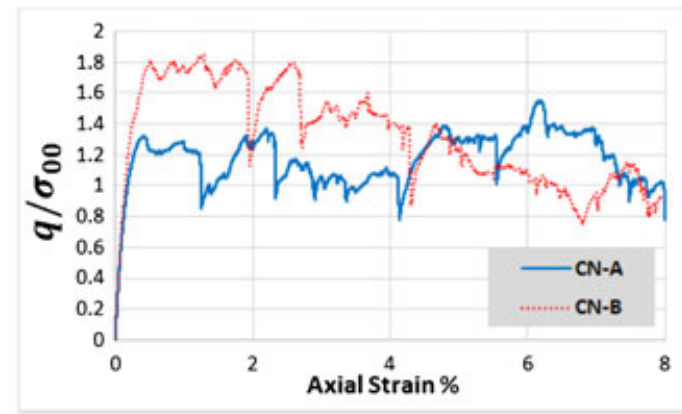

(b)

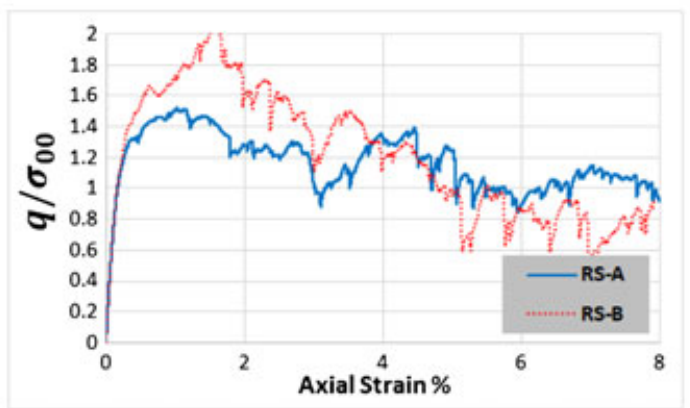

(c)

Figure 5. Pure DEM simulations of monotonic compression biaxial test up to $8 \%$ axial strain performed on six different DEM assemblies of identical material properties but different with respect to geometry (different particles positions and grain size distribution). (a) PF-A and PF-B; (b) CN-A and CN-B; and (c) RS-A and RS-B.

stress is computed as $q=\sigma_{1}-\sigma_{2}$, where $\sigma_{1}$ and $\sigma_{2}$ are the vertical normal stress and the lateral normal stress, respectively. The obtained results show that all cases exhibit, after a pre-peak smooth response, global strength loss, which is a characteristic of strain softening behavior. The strong fluctuations observed in the post-peak part of the curves can be attributed to the relatively small number of particles (400 particles) that leads to a series of sudden contact rearrangements along the deforming process. Despite these differences in the strength peak, all assemblies tend to a similar level of residual strength (plateau around $q / \sigma_{00}=0.9$ ).

Though the six assemblies have the same DEM mechanical parameters (Table II) and differ only with the random geometric factors (described in Section 3.3), the pure DEM simulations show that they have different strength peaks; the resultant normalized deviatoric strength peaks vary from 1.3 to 1.8. This divergence in the granular assemblies behavior comes only from their geometry differences. Subsequently, these observations show that geometry differences have strong effects on the mechanical properties of the granular assembly.

\section{2. $F E M \times D E M$ simulation}

Each REV is then used for the initially homogeneous FEM $\times$ DEM simulations. The numerical specimen is subjected to a biaxial loading path up to $8 \%$ axial strain. Figure 6 shows the global response of the six FEM $\times$ DEM specimens compared with the pure DEM simulations. In this figure, all the cases show that each FEM $\times$ DEM specimen has the same mechanical response as the associated DEM REV up to the strength peak. In contrast, the post-peak response of FEM $\times$ DEM specimens show a clear divergence from the DEM simulation. Indeed, beyond the peak, strain localisation starts to develop, which explains the divergence from material behavior. Moreover, while the response of the DEM assembly strongly fluctuates, the FEM $\times$ DEM specimen exhibits a smoother response. This can be attributed to the averaging effect of the integration by the FEM numerical process of the inherently noisy responses of the different REVs involved in the structure, especially those 

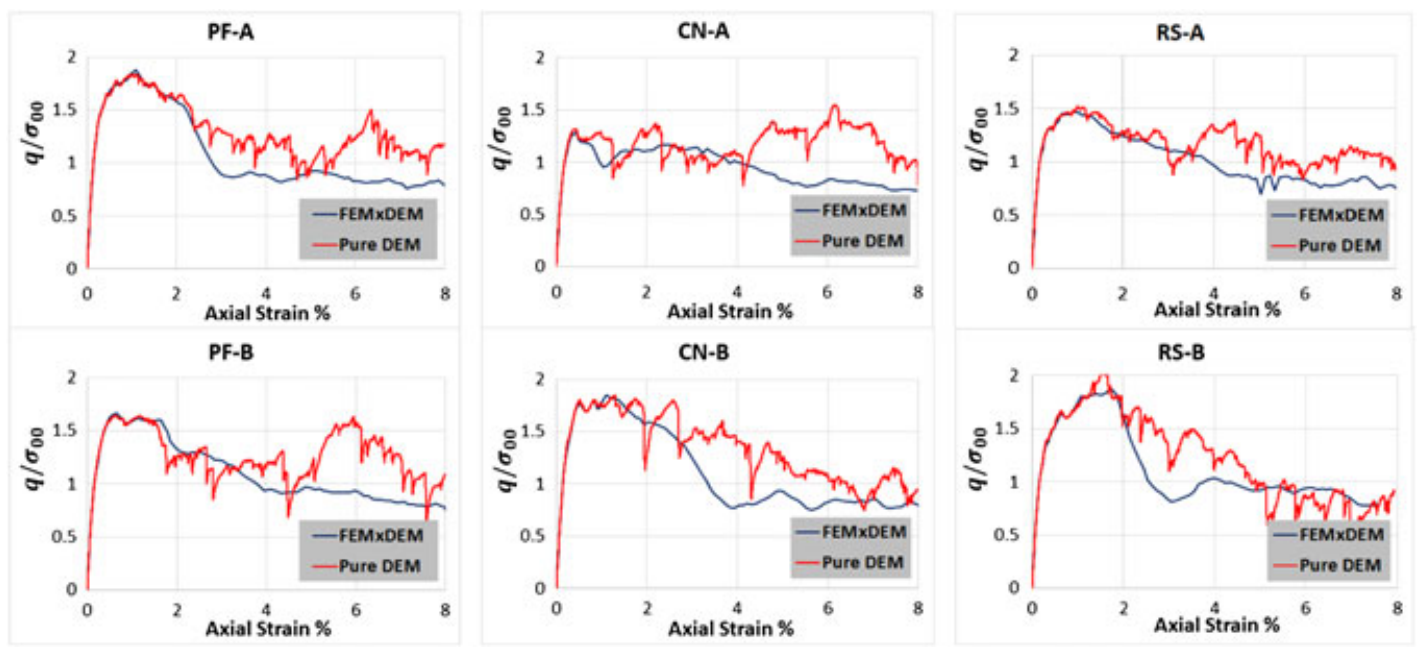

Figure 6. A comparison between the global responses of the homogeneous FEM $\times$ DEM simulations with the pure DEM simulations of the corresponding DEM REV in biaxial test simulations up to $8 \%$ axial strain.

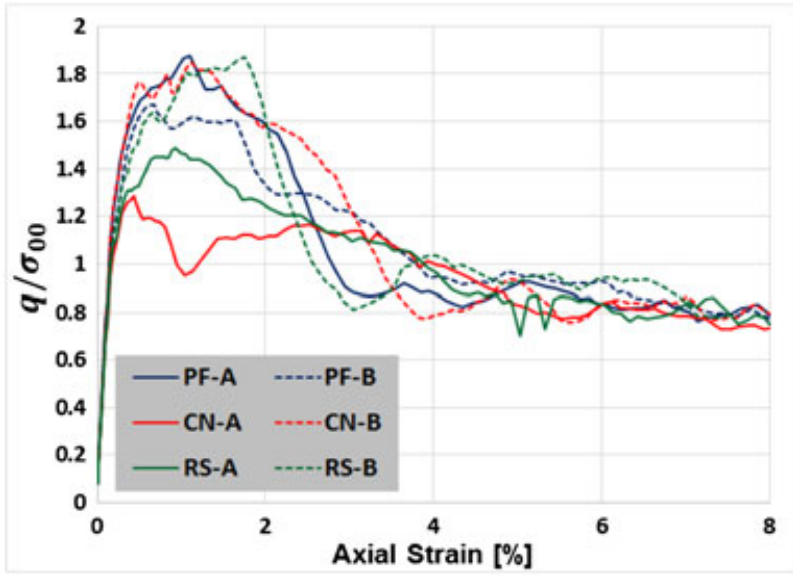

Figure 7. Global responses of six homogeneous specimens in a biaxial test up to $8 \%$ axial strain simulations. The used DEM REVs, for microscale, are identical with respect to material properties but different concerning the geometry.

associated to the Gauss points that lie in the shear band zone. At the end of the test, the FEM $\times$ DEM specimen and the DEM assembly tend to a similar residual strength plateau.

Figure 7 compares the six FEM $\times$ DEM simulations. It can be observed that even with different strength peaks, all the FEM $\times$ DEM simulations tend to a similar residual strength level (around $\left.q / \sigma_{0}=0.9\right)$. This result is consistent with what is classically observed in DEM modeling: for large strain, the mechanical behavior of granular material is mainly ruled by the particle shapes and the inter-granular angle of friction [34].

\section{INHOMOGENEOUS MULTISCALE MODELING}

So far, a single DEM assembly has been used in each FEM $\times$ DEM simulation. The use of a unique DEM REV over the entire mesh implies a uniform representation of the microstructure and leads to an initially homogeneous specimen. However, the experimental evidence shows that a geomaterial sample presents a variety of microscopic structures (Figure 1). The differences of the microstructure in heterogeneous geomaterials result in a variability of the local mechanical properties, which might play a major role in the resulting global behavior and the formation of the shear band. This part of the work is devoted to this particular issue, which is the motivation of the whole study. 


\subsection{Inhomogeneous simulations setup}

The FEM $\times$ DEM approach naturally offers the possibility of taking into account the heterogeneous nature of geomaterials. The variation of the local mechanical properties, originating from the microstructure, is then introduced into an FEM $\times$ DEM simulation by using several DEM assemblies, representative of the same material but with geometrical differences (Table III). This approach allows to enhance the original model by embedding heterogeneities, while the average material properties are the same. To simplify this investigation, the heterogeneity has been introduced in the following FEM $\times$ DEM simulations by using two different DEM REVs. The way the two DEM REVs interplay with the macroscale continuum is investigated hereafter.

Based on the selection criteria introduced in Section 3.3, the set of the six DEM REVs was split into three groups: PF group (PF-A and PF-B), $\mathrm{CN}$ group (CN-A and CN-B), and RS group (RS-A and RS-B). Each group is referred as PFG, CNG, and RSG, respectively. In Table V, the granular assemblies in each group were distinguished into strong and weak REVs according to their strength peak (Section 5.1). The granular assembly with the higher strength peak is referred as strong REV (i.e., PF-A, CN-B, and RS-B), whereas the assembly with the lower strength peak is referred as weak REV (i.e., PF-B, CN-A, and RS-A) (Figure 5).

Each pair of these REVs is then used in different FEM $\times$ DEM simulation. The in-plane distribution of the two DEM REVs is performed following two different patterns: a checkerboard pattern and a random distribution pattern (Figure 8). Consequently, the six inhomogeneous specimens have been used to run a set of biaxial test simulations up to $8 \%$ axial strain. Each FEM $\times$ DEM simulation will be identified by the name of the associated REVs group, namely, PFG, CNG, and RSG.

\subsection{Results and discussion}

Figure 9 shows the results obtained from PFG simulations with the checkerboard pattern (C-PFG) and the random distribution pattern (R-PFG). Figure 9(a) shows the global response of these inhomogeneous specimens. The global response of the corresponding homogeneous specimens, PF-A and PF-B, are presented in the same figure. This comparison shows that the inhomogeneous specimens yield to identical pre-peak responses. In both cases, the strength peak of the inhomogeneous specimen has been determined by the strength capacity of the weak REV (PF-B). Furthermore, the inhomogeneous specimens show a clear divergence in the strain softening behavior; however, they tend to a similar residual strength. Figure 9(b) and (c) displays the cumulative deviatoric strain field

Table V. The six DEM assemblies split into three different subsets. The dark gray and the light gray refer to the strong and the weak REVs, respectively.
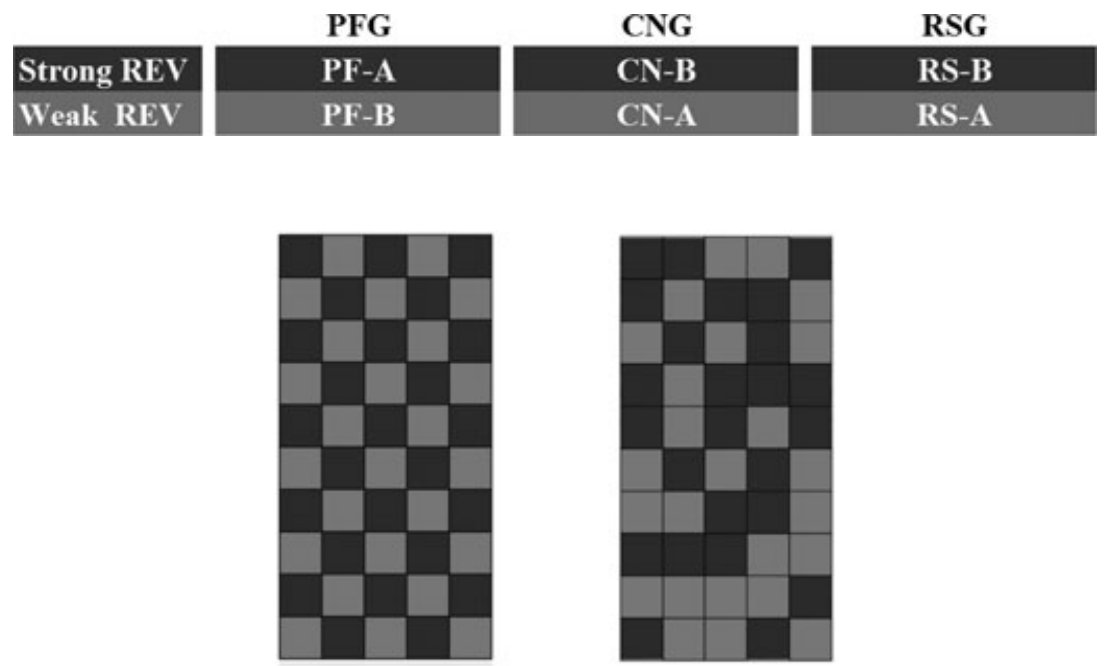

Figure 8. The checkerboard pattern and the random distribution pattern of the inhomogeneous specimens. The dark gray refers to the elements that were associated with the strong REV, whereas the light gray refers to the elements that were associated with the weak REV. 


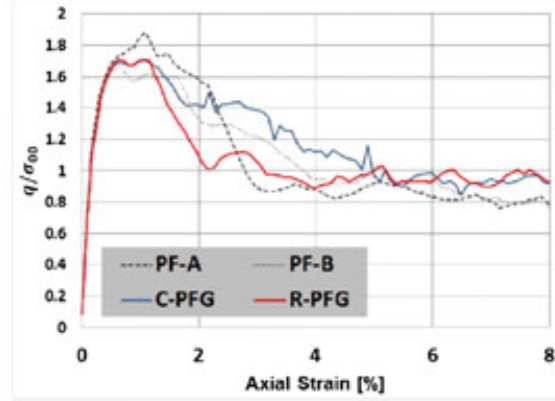

(a)

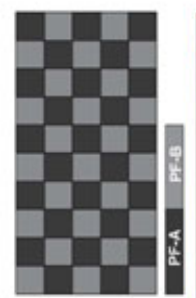

(b)

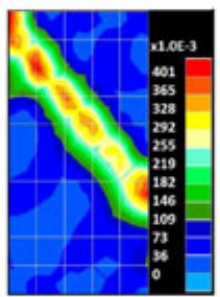

(c)

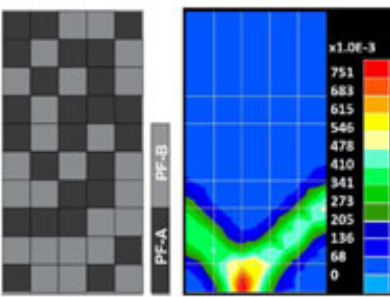

Figure 9. (a) The global response of the inhomogeneous specimens in which PF-A and PF-B have been attached in the dark gray and light gray elements, respectively, following the checkerboard pattern (blue) and the random distribution pattern (red). The global response of the homogeneous specimens corresponding to the used DEM REVs are presented as well. (b) and (c) The cumulative deviatoric strain field corresponding to the specimen of checkerboard pattern and random distribution pattern inhomogeneity, respectively, at the end of the test.

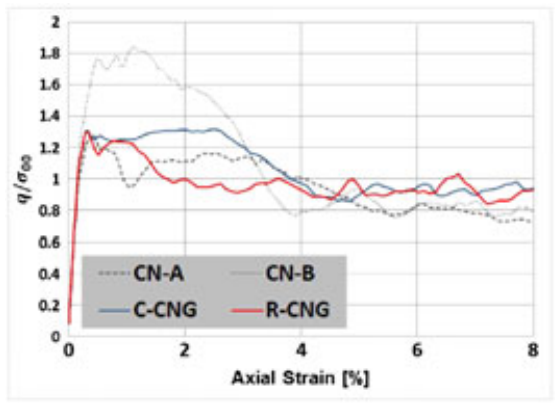

(a)

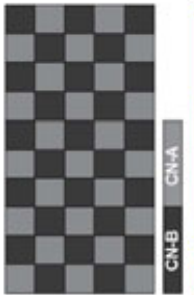

(b)
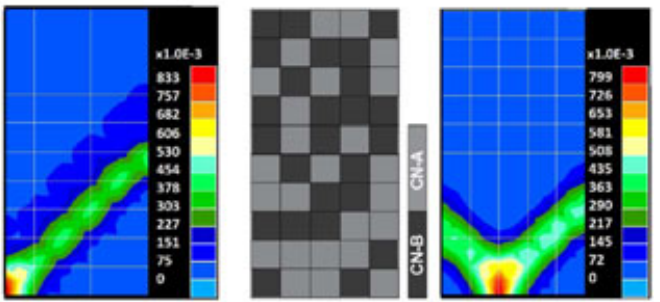

(c)

Figure 10. (a) The global response of the inhomogeneous specimen in which $\mathrm{CN}-\mathrm{A}$ and $\mathrm{CN}-\mathrm{B}$ have been attached in the dark gray and light gray elements, respectively, following the checkerboard pattern (blue) and the random distribution pattern (red). The global response of the homogeneous specimens corresponding to the used DEM REVs are presented as well. (b) and (c) The cumulative deviatoric strain field corresponding to the specimen of checkerboard pattern and random distribution pattern inhomogeneity, respectively, at the end of the test.

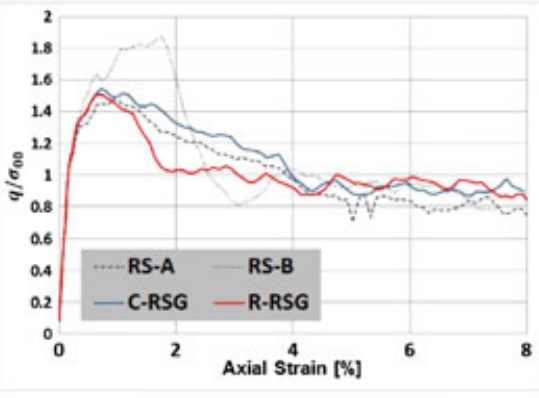

(a)

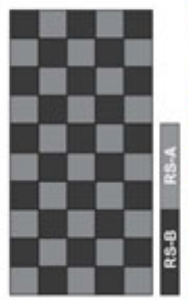

(b)
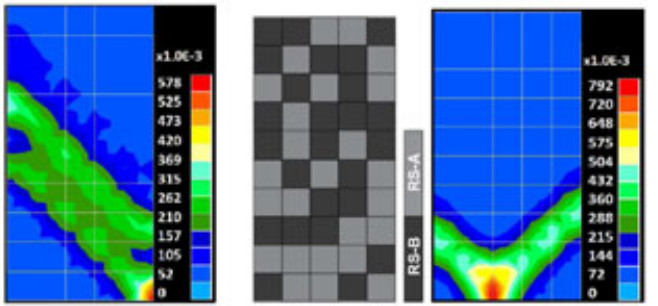

(c)

Figure 11. (a) The global response of the inhomogeneous specimen in which RS-A and RS-B have been attached in the dark gray and light gray elements, respectively, following the checkerboard pattern (blue) and the random distribution pattern (red). The global response of the homogeneous specimens corresponding to the used DEM REVs are presented as well. (b) and (c) The cumulative deviatoric strain field corresponding to the specimen of checkerboard pattern and random distribution pattern inhomogeneity, respectively, at the end of the test. 
at $8 \%$ axial strain (the end of the simulation) of the specimens with the checkerboard pattern and the random distribution pattern, respectively. In Figure 9(b), it should be noted that the shear band, although it may seem to reach the left boundary of the specimen at the upper-left corner, is in fact essentially aligned with the line of light gray elements below this corner. As a result, both cases demonstrate that the strain field has localized in the elements that were associated with the weak REV (PF-B).

The results obtained from the CNG simulations with the checkerboard pattern $(\mathrm{C}-\mathrm{CNG})$ and the random distribution pattern (R-CNG) are presented in Figure 10. These results confirm the aforementioned observations regarding the identical pre-peak response and the dominant role of the weak REV (CN-A) at defining the strength peak of the inhomogeneous specimen. The great dispersion of the mechanical behavior of the REVs, used in this set of experiments (Figure 5), provides more pronounced evidence of such role. Also, they corroborate the role of the weak REV at the definition of the shear band pattern.

Figure 11 shows the results of the third set of experiments in which the RSG assemblies were retained. The global response of the inhomogeneous specimens compared with the corresponding homogeneous simulations are presented in Figure 11(a). This comparison confirms the observations made earlier concerning the capability of the weak REV (RS-A) at defining the strength peak of the inhomogeneous specimen. Also, it confirms that, after a divergent strain softening behavior, the inhomogeneous specimen shows a similar residual strength level. Figure 11(b) and (c) displays the cumulative deviatoric strain field at $8 \%$ axial strain of the inhomogeneous specimens of checkerboard pattern and random distribution pattern, respectively. Both cases have demonstrated similar observations regarding the shear band pattern that is determined by an alignment of elements attached with the weak REV. Moreover, another feature of the shear band pattern has been obtained in the simulation with checkerboard inhomogeneity: the strain field has localized in two different shear bands reflecting the periodicity of inhomogeneity.

The homogeneous simulations of both CNG and RSG have shown clear differences in the strain softening behavior; while the homogeneous CN-B and RS-B simulations exhibit brittle behavior, the $\mathrm{CN}-\mathrm{A}$ and RS-A simulations are rather ductile. However, the results obtained from the CNG and RSG simulations set reveal that the inhomogeneous models tend to exhibit a more ductile behavior. This matter can be attributed to the role of the weak REV, which is of more ductile behavior, at triggering strain localization and then defining the shear band pattern in the inhomogeneous simulation.

As a conclusion, the use of two different DEM REVs for the microstructure representation helps the specimen to define its shear band pattern that develops at an alignment of elements associated with the weak REV. Moreover, the weak REV triggers strain localization and consequently plays a major role in the definition of the specimen strength peak.

\section{MATERIAL IMPERFECTION SIMULATION}

The capability of using several REVs to capture the intrinsic heterogeneity of geomaterials have been explored in the previous section. In what follows, a material imperfection, represented by the weak REV, is introduced into homogenous simulation.

Experimental evidence show that introducing material imperfection into soil specimen facilitates triggering strain localization and then this imperfection becomes a crucial path of the consequent shear band [32]. The efficiency of simulating this experiment will be investigated using the weak REV to introduce material imperfection into homogeneous specimen. The granular assemblies set, $\mathrm{CNG}$, has been chosen considering that $\mathrm{CN}-\mathrm{A}$ and $\mathrm{CN}-\mathrm{B}$ have shown the greatest dispersion in the mechanical properties (strength peak) (Figure 5(b)). The imperfection is introduced into the specimen by changing the DEM REV in a single element. The remaining 49 elements are associated with the CN-B. Figure 12(b) depicts the distribution of the DEM REVs over the FE mesh. Hereafter, this specimen is referred as IMP. As in the previous cases, the behavior of the IMP has been investigated through a biaxial test simulation up to $8 \%$ axial strain. Figure 12(a) shows the global response of the IMP specimen; the global responses of the corresponding homogeneous specimens, $\mathrm{CN}-\mathrm{A}$ and $\mathrm{CN}-\mathrm{B}$, have been presented in the same figure as well. 


\section{G. SHAHIN ET AL}

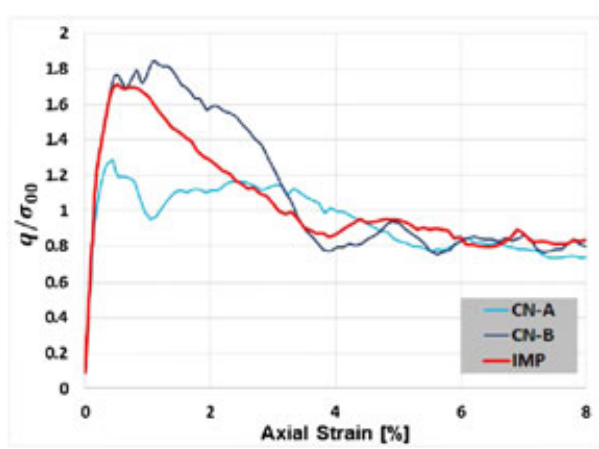

(a)

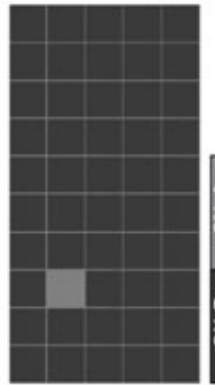

(b)

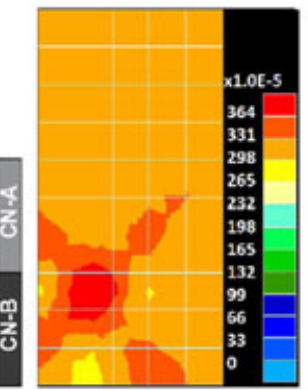

(c)

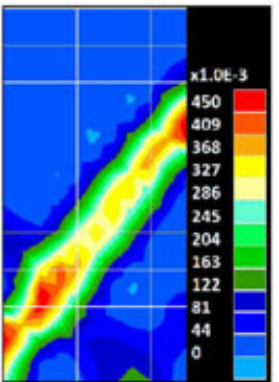

(d)

Figure 12. A comparison between homogeneous specimen and a specimen that have been introduced material imperfection by attaching an REV of weaker strength peak at a single element: (a) compares the global responses of the imperfect specimen (IMP) and the corresponding homogeneous specimens of the weak and the strong REVs and (b) shows the position of the element, which is associated with the weak REV (light gray). The cumulative deviatoric strain field corresponds to (c) $0.35 \%$ and (d) $8.0 \%$ axial strain.

Table VI. The relative time cost of the homogeneous CN-B simulation and IMP simulation.

\begin{tabular}{lc}
\hline Specimen & Time cost [\%] \\
\hline Homogeneous CN-B specimen & 100 \\
IMP specimen & 68 \\
\hline
\end{tabular}

Comparing the IMP response with the homogeneous simulations shows that, after an identical pre-peak behavior, the IMP yield to a smoother response at and beyond the peak. The weak REV has not affected the strength capacity of IMP specimen, as the slight reduction in the strength peak can be neglected comparing with the corresponding inhomogeneous simulations in the previous section.

Figure 12(c) and (d) displays the cumulative deviatoric strain field corresponding to $0.35 \%$ and $8 \%$ axial strain, respectively. As anticipated, it can be observed that the element associated with the weak REV (CN-A) has triggered strain localization at $0.35 \%$ axial strain. Moreover, the consequent shear band passes through the imperfect element as can be seen in Figure 12(d).

It is worthy to mention that the introduction of material imperfection into simulation specimen has a positive effect on the computation time. Table VI displays the time cost associated to both the IMP and the homogeneous CN-B simulations. The introduction of material imperfection has reduced time cost about $35 \%$. This matter can be attributed to the capability of the imperfect element to trigger strain localization and to define the shear band locus along the deformation process. This issue has been extensively studied in [35]. These observations give a first hint to the capability of using different DEM REVs with the aforementioned specifications to improve the stability of the FEM $\times$ DEM simulations.

\section{CONCLUSION}

The consequences of using several DEM REVs for the microscale simulation in the framework of multiscale FEM $\times$ DEM modeling approach have been investigated in this work. The DEM assemblies were generated having the same material properties but with geometrical alterations. These geometrical alterations have a strong effect on the mechanical behavior of the granular assembly as shown by the pure DEM simulations. The performed homogeneous FEM $\times$ DEM simulations have shown a similar effect of geometry alteration, but with a much smoother response.

The performed inhomogeneous FEM $\times$ DEM simulations reveal a clear evidence of the capability of the weak DEM REV to trigger strain localization and to help the specimen at defining its shear band pattern. 
Using a weak DEM REV to introduce local imperfection into homogeneous FEM $\times$ DEM specimen has shown a satisfactory effectiveness in terms of triggering strain localization; indeed, the consequent shear band pattern passes through the imperfect element. The obtained results have given a first hint to the ability of this technique to improve the stability of the problem and then to reduce time cost.

In future work, it is worth to investigate the consequences of using a larger number of DEM REVs in the FEM $\times$ DEM simulations. A FE mesh of 50 elements has been used for the macroscale level, and it is of significant importance to reevaluate the findings of this work with a finer FE mesh. Also, it is recommended to repeat this study, adopting a regularization technique such as the secondgradient theory, in order to mitigate the well-known effect of mesh dependency; this issue was out of the scope of this study.

\section{APPENDIX A: SENSITIVITY ANALYSIS}

In this part, a sensitivity analysis is proposed to define a set of four parameters among others used in the numerical FEMxDEM analysis. The four parameters are the inertial number (IN) and the level of equilibrium (LE) conditions that are relevant to DEM scheme and the perturbation magnitude (PM) and the precision level $\left(E_{F}\right.$ and $\left.E_{D}\right)$ that are relevant to FEM scheme. These four parameters were selected assuming that they have an influential role on the simulation results and time cost. The numerical experiments were carried out on two different FEM $\times$ DEM simulations: the first was homogeneously made of CN-A as an REV and the second was the RS-A.

\section{A.1. DEM parameters: the inertial number and the level of equilibrium conditions}

The IN is a physical parameter related to DE simulations that describes the strain-rate applied on the granular assembly. As proposed in $[10,11]$, the quasi-static conditions are satisfied if the IN $\leqslant 1.0$ E-3. Reducing the IN magnitude improves the quasi-static conditions; however, this reduction significantly increases time cost. Therefore, two different values of the IN have been examined: $\mathrm{IN}=1.0 \mathrm{E}-3$ and $1.0 \mathrm{E}-4$. The LE parameter is the second DEM parameter that affects the quality of the solution. The requirement of a higher LE conditions improves both the stability of the problem and the quality of the solution; however, this leads to a significant increase in the computational time. The LE parameter was examined through two different levels, LE $=2$ and 4 considering the six levels of equilibrium conditions implemented in DEM code (Table I). The values of these two parameters have been combined in four different combinations that are used in a set of biaxial test simulations up to $2 \%$ axial strain. Table A.1 shows the four combinations and the associated time cost.

Figure A.1(a) and (b) shows the global response of the two specimens corresponding to each combination. Simulation number 1 combines the parameters with the stronger values (i.e., these leading to higher quality of DEM modeling). Therefore, this simulation has been considered as a

Table A.1. The different combinations of parameters defined for the inertial number and the level of equilibrium conditions sensitivity study and the corresponding relative time cost, for $2 \%$ axial strain.

\begin{tabular}{|c|c|c|c|c|c|c|c|}
\hline \multirow{2}{*}{ No. } & \multirow{2}{*}{ IN } & \multirow{2}{*}{ LE } & \multirow{2}{*}{ PM } & \multirow{2}{*}{$\mathrm{ED}$} & \multirow{2}{*}{$\mathrm{EF}$} & \multicolumn{2}{|c|}{ Time cost [\%] } \\
\hline & & & & & & $\mathrm{CN}-\mathrm{A}$ & RS-A \\
\hline 1 & & 4 & \multirow{4}{*}{$15.0 \mathrm{E}-6$} & \multirow{4}{*}{0.01} & \multirow{4}{*}{0.01} & 100 & 67 \\
\hline 2 & $1.0 \mathrm{E}-4$ & 2 & & & & 81 & 61 \\
\hline 3 & \multirow{2}{*}{$1.0 \mathrm{E}-3$} & 4 & & & & 88 & 100 \\
\hline 4 & & 2 & & & & 62 & 52 \\
\hline
\end{tabular}

IN, inertial number; LE, level of equilibrium; PM, perturbation magnitude; $\mathrm{ED}$, displacement-based convergence criterion; EF, force-based convergence criterion. 


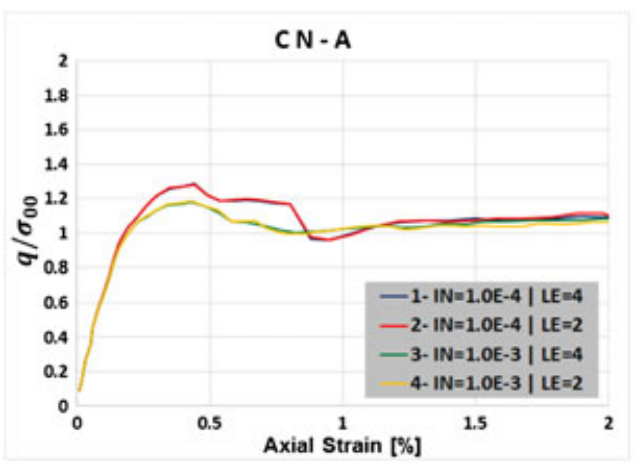

(a)

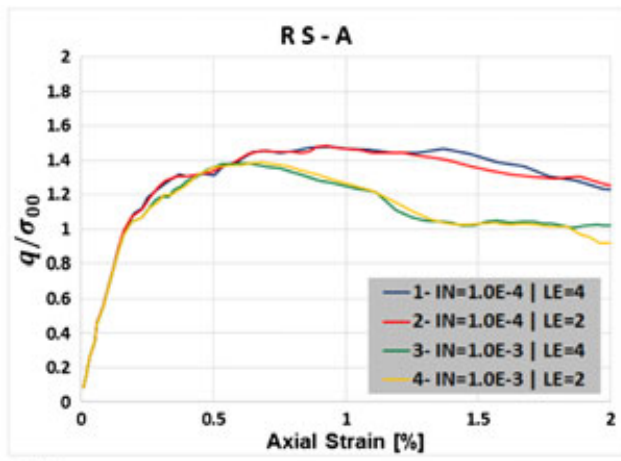

(b)

Figure A.1. A comparison of the global responses of the two specimens (a) CN-A and (b) RS-A corresponding to four different combinations of the IN and the LE conditions, for $2 \%$ axial strain.

reference case. All cases have shown quite comparable pre-peak responses. However, simulations numbers 3 and 4 show a softer response in the post-peak domain. These two simulations share the same value of the IN, IN $=1.0 \mathrm{E}-3$, which refers to an important effect of this parameter on the mechanical behavior of the FEM $\times$ DEM specimen, specifically in the post-peak domain. In contrast, simulation number 2 has demonstrated a good agreement in the behavior and a reduction in time cost about $10 \%$ comparing to reference case. Therefore, the values of the IN and the LE in combination number 2 have been adopted hereafter.

\section{A.2. FEM parameters: the perturbation magnitude and the precision level}

A.2.1. Perturbation magnitude. The derivation of the CTO relies on the computation of the REV response on perturbed paths close to the actual incremental strain paths. The effects of the perturbation magnitude on analysis results will be explored hereafter.

Loading is applied on the specimen as a vertical displacement of the top horizontal boundary nodes. At the macro scale level (FEM), loading process is driven using an adaptive scheme, which means that the displacement increment applied at each loading step (hereafter called 'step size') might change during the computation. Therefore, a lower bound has to be specified for the step size in order to avoid unlimited step size reduction in case of non convergence. This bound was given the value $5.0 \mathrm{E}-5$, which is equivalent to $2.5 \mathrm{E}-5$ vertical strain. The determination of the strain perturbation magnitude has to consider that the PM in the numerical derivation of the CTO has to be defined as a fraction of the local strain rate increment, which depends on the adaptive step size. Consequently, the lower bond of step size has been considered as a reference value, and three values of PM have been examined: 5.0E-6, 10.0E-6, and 15.0E-6, which are fractions of the lower bound. These values were used in the three combinations shown in Table A.2, in a set of biaxial test simulations up to $0.4 \%$ axial strain (found to require 15 steps) using both specimens $\mathrm{CN}-\mathrm{A}$ and RS-A.

Figure A.2(a) and (b) displays the global responses of CN-A and RS-A specimens, respectively. The three cases have led to comparable results with negligible changes in time cost. Such negligible

Table A.2. The three combinations defined for perturbation magnitude sensitivity study.

\begin{tabular}{lccrcc}
\hline No. & IN & LE & \multicolumn{1}{c}{ PM } & ED & EF \\
\hline 5 & & & $5.0 \mathrm{E}-6$ & & \\
6 & $1.0 \mathrm{E}-4$ & 2 & $10.0 \mathrm{E}-6$ & 0.01 & 0.01 \\
7 & & & $15.0 \mathrm{E}-6$ & & \\
\hline
\end{tabular}

$\overline{\mathrm{IN}}$, inertial number; LE, level of equilibrium; PM, perturbation magnitude; ED, displacement-based convergence criterion; $\mathrm{EF}$, force-based convergence criterion. 


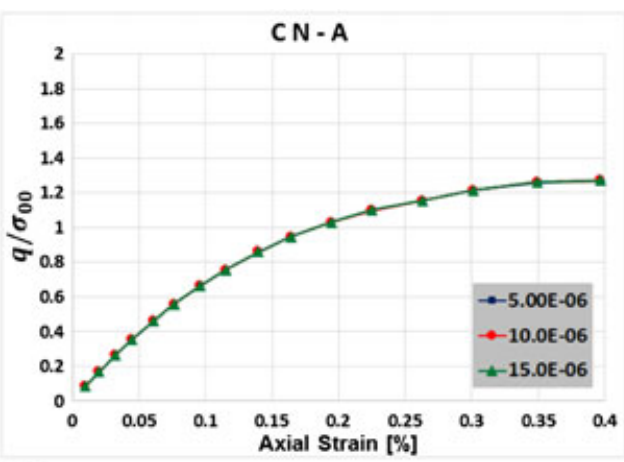

(a)

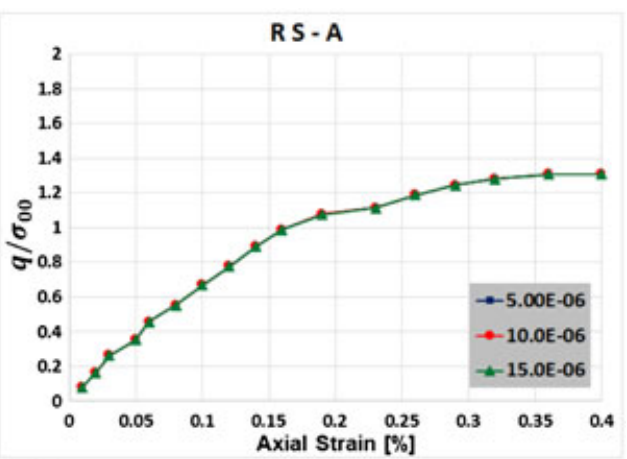

(b)

Figure A.2. A comparison of the global responses of the two specimens (a) CN-A and (b) RS-A corresponding to three different PM: 5.0E-6, 10.0E-6, and $15.0 \mathrm{E}-6$, for $0.4 \%$ axial strain.

Table A.3. The different combinations of technical parameters defined for precision level $\left(E_{F}\right.$ and $\left.E_{D}\right)$ sensitivity study and the corresponding relative time cost, for $8 \%$ axial strain.

\begin{tabular}{lccccccc}
\hline No. & IN & LE & PM & ED & EF & \multicolumn{2}{c}{ Time cost [\%] } \\
\cline { 5 - 7 } & & & & & CN-A & RS-A \\
\hline 7 & \multirow{2}{*}{$1.0 \mathrm{E}-4$} & 2 & $15.0 \mathrm{E}-6$ & 0.01 & 0.02 & 100 & 100 \\
\hline
\end{tabular}

IN, inertial number; LE, level of equilibrium; PM, perturbation magnitude; $\mathrm{ED}$, displacement-based convergence criterion; $\mathrm{EF}$, force-based convergence criterion.

changes in time cost can be attributed to the fact that perturbation method is applied in the first steps when material behavior is quasi-elastic. Consequently, this parameter has a negligible influence on the solution and thus a PM equal to $15.0 \mathrm{E}-6$ has been arbitrarily chosen to be used in this work.

A.2.2. The precision level. The quality of the solution at the FEM scale is controlled by the precision parameters (convergence criteria) of the integration scheme. The required quality of the solution is specified by two parameters: a force-based parameter, $E_{F}$, representing the ratio of the applied and the reaction forces norm, together with a displacement-based parameter, $E_{D}$, accounting for the decrement of the displacement field norm with the evolution of the iterative process [28].

Guo and Zhao [20] reported the use of displacement-based criterion solely as a convergence indicator with $E_{D} \leqslant 0.01$ for a similar multiscale $\mathrm{FEM} \times \mathrm{DEM}$ simulation. This assumption was based on a common consideration in the commercial FEM software such as GEO5, ANSYS, and ABAQUS. In this study, two different precision combinations were defined: $E_{D}\left|E_{F}=0.01\right| 0.02$ and $0.01 \mid$ NA (i.e., double convergence criteria with $E_{D}=0.01$ and $E_{F}=0.02$, and single convergence criterion with $E_{D}=0.01$ disregarding the $E_{F}$ criterion). In the sequel, the former is referred as higher precision combination (HPC) and the latter lower precision combination (LPC). These two combinations were used in several biaxial test simulations up to $8 \%$ axial strain on both $\mathrm{CN}-\mathrm{A}$ and RS-A specimens. Table A.3 depicts the performed simulations and the associated time cost.

Figure A.3 shows the global responses of both simulations, CN-A and RS-A, with HPC and LPC. Generally speaking, no important change can be observed in the global responses of the specimens when the force-based criterion is considered together with the displacement-based criterion. However, neglecting the force-based criterion induces a significant reduction, about $75-80 \%$, in time cost. 


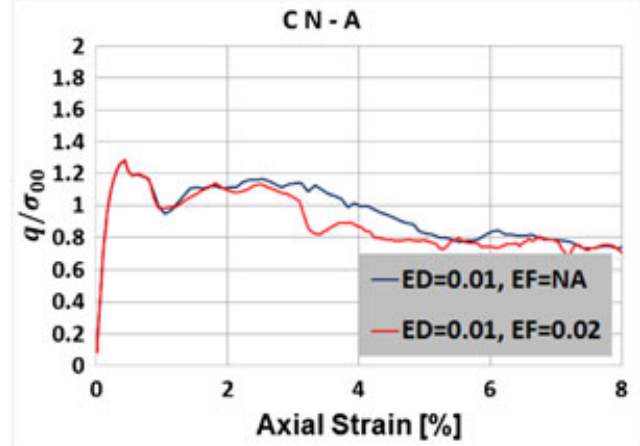

(a)

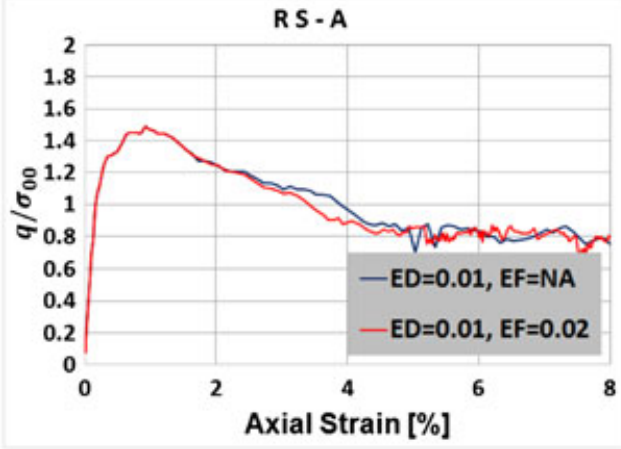

(b)

Figure A.3. A comparison of the global responses of two specimens (a) CN-A and (b) RS-A at two different precision combinations: $E_{D}\left|E_{F}=0.01\right| \mathrm{NA}$ and $0.01 \mid 0.02$, for $8 \%$ axial strain; ED, displacement-based convergence criterion; $\mathrm{EF}$, force-based convergence criterion.

Table A.4. The adopted computational parameters values based on the performed sensitivity study.

\begin{tabular}{cccc}
\hline $\mathrm{IN}$ & $\mathrm{LE}$ & $\mathrm{PM}$ & $\mathrm{ED} \mid \mathrm{EF}$ \\
\hline $1.0 \mathrm{E}-4$ & 2 & $15.0 \mathrm{E}-6$ & $0.01 \mid \mathrm{NA}$ \\
\hline
\end{tabular}

$\overline{\mathrm{IN}}$, inertial number; LE, level of equilibrium; PM, perturbation magnitude; ED, displacement-based convergence criterion; EF, force-based convergence criterion.

As a result of this sensitivity analysis, the four parameters that are used in the current work are defined and summarized in Table A.4.

\section{ACKNOWLEDGEMENTS}

Ghassan Shahin acknowledges the financial support of the European Education, Audiovisual and Culture Executive Agency (EACEA), under the Erasmus Mundus Master in Earthquake Engineering and Engineering Seismology (MEEES) program. Also, he is pleased to acknowledge with thanks the helpful discussions with Abraham Van den Eijnden. Laboratory 3SR is a part of the LabEx Tec 21 (Investissements d'Avenir grant agreement no. ANR-11-LABX-0030).

\section{REFERENCES}

1. Kaneko K, Terada K, Tyoya T, Kishino Y. Global-local analysis of granular media in quasi-static equilibrium. International Journal of Solids and Structures 2003; 40(15):4043-4069.

2. Meier H, Steinmann P, Kuhl E. On the multiscale computation of confined granular media. In ECCOMAS Multidisciplinary Jubilee Symposium, Computational Methods in Applied Sciences, Vol. 14, Eberhardsteiner J, Hellmich C, Mang H, Priaux J (eds). Springer: Netherlands, 2009; 121-133.

3. Miehe C, Dettmar J, Zah D. Homogenization and two-scale simulations of granular materials for different microstructural constraints. International Journal for Numerical Methods in Engineering 2010; 83(8-9):1206-1236.

4. Nitka M, Combe G, Dascalu C, Desrues J. Two-scale modeling of granular materials: a DEM-FEM approach. Granular Matter 2011; 13(3):277-281.

5. Andrade J, Avial C, Hall S, Lenoir N, Viggiani G. Multiscale modelling and characterization of granular matter: from grain kinematics to continuum mechanics. Journal of the Mechanics and Physics of Solids 2011; 59(2):237-250.

6. Guo N, Zhao J. A hierarchical model for cross-scale simulation of granular media. AIP Conference Proceedings 2013; 1542(1):1222-1225.

7. Wellmann C, Lillie C, Wriggers P. Homogenization of granular material modeled by a three-dimensional discrete element method. Computers and Geotechnics 2008; 35(3):394-405.

8. Geers M, Kouznetsova V, Brekelmans W. Multi-scale computational homogenization: trends and challenges. Journal of Computational and Applied Mathematics 2010; 234(7):2175-2182. Fourth International Conference on Advanced COmputational Methods in ENgineering (ACOMEN 2008). 
9. Cundall P, Ruest M, Guest A, Chitombo G. Evaluation of shemes to improve the efficiency of a complete model of blasting and rock fractures. In Numerical Modeling in Micromechanics via Particles Methods, (Proceedings of the 1st International PFC Symposium), Konietzky H (ed.). Balkema: Gelsenkirchen, Germany, 2003; 107-115.

10. Radjai F, Dubois F. Discrete-element Modeling of Granular Materials. Wiley, 2011.

11. Roux J, Combe G. How granular materials deform in quasistatic conditions. In IUTAM-ISIMM Symposium on Mathematical Modeling and Physical Instances of Granular Flows, AIP Conference Proceedings, Vol. 1227. Reggio Calabria: Italy, 2010; 260-270.

12. Cundall P. A discontinuous future for numerical modelling in geomechanics? Proceedings of the ICE-Geotechnical Engineering 2001; 149(1):41-47.

13. Terada K, Kurumatani M. Two-scale diffusion-deformation coupling model for material deterioration involoving micro-crack propagation. International Journal for Numerical Methods in Engineering 2010; 83(4):426-451.

14. Miehe C, Dettmar J. A framework for micro-macro transitions in periodic particle aggregates of granular materials. Computer Methods in Applied Mechanics and Engineering 2004; 193(35):225-256.

15. Meier H, Steinmann P, Kuhl E. Towards multiscale computation of confined granular media. Technische Mechanik 2008; 28(1):32-42.

16. Avial C, Andrade J. Advances in multiscale modelling and characterization of granular matter. Procedia IUTAM 2012; 3(0):157-171.

17. Nitka M, Bilbie B, Combe G, Dascalu C, Desrues J. A micro-macro (DEM-FEM) model of the behavior of granular solids. In 1st International Symposium on Computational Geomechanics (ComGeo I), Juan-les-Pins, France, 2009; $38-48$.

18. Nguyen TK, Combe G, Caillerie D, Desrues J. Modeling of a cohesive granular materials by a multi-scale approach. AIP Conference Proceedings, Vol. 1542, Sydney, Australia, 2013; 1194-1197.

19. Nguyen T, Combe G, Caillerie D, Desrues J. FEM $\times$ DEM modelling of cohesive granular materials: numerical homogenisation and multi-scale simulations. Acta Geophysica 2014; 62(5):1109-1126.

20. Guo N, Zhao J. A coupled FEM/DEM approach for hierarchical multiscale modelling of granular media. International Journal for Numerical Methods in Engineering 2014; 99(11):789-818.

21. Desrues J, Nguyen T, Combe G, Caillerie D. FEM $\times$ DEM multi-scale analysis of boundary value problems involving strain localization. In Bifurcation and Degradation of Geomaterials in the New Millennium, Chau KT, Zhao J (eds)., Springer Series in Geomechanics and Geoengineering. Springer International Publishing, 2015; 259-265.

22. Zhao J, Guo N. The interplay between anisotropy and strain localisation in granular soils: a multiscale insight. Géotechnique 2015; 65(8):642-656.

23. Guo N, Zhao J. Multiscale insights into classical geomechanics problems. International Journal for Numerical and Analytical Methods in Geomechanics 2015. DOI: 10.1002/nag.2406, In press.

24. Andò E. Étude experimentale de l'évolution de la microstructure d'un milieu granulaire sous chargement mécanique a l'aide de la tomographie rayons x. PhD Thesis, Docteur de L'Université de Grenoble, 2013.

25. Weber J. Recherches concernant les contraintes intergranulaires dans les milieux pulverulents, Bull. Liaison des Ponts et Chaussees 1966; 22:1-20.

26. Coenen E, Kouznetsova V, Bosco E, Geers M. A multi-scale approach to bridge microscale damage and macroscale failure: a nested computational homogenization-localization framework. International Journal of Fracture 2012; 178(1-2):157-178.

27. Bosco E, Kouznetsova VG, Geers MGD. Multi-scale computational homogenization-localization for propagating discontinuities using X-FEM. International Journal for Numerical Methods in Engineering 2015; 102(3-4):496-527.

28. Charlier R. Approache unifiée, de quelques problémes non linéaires de mécanique des milieux continus par la méthode des éléments finis. PhD Thesis, University of Liège, France, 1987.

29. Zienkiewicz O, Taylor R, Zhu J. The Finite Element Method: Its Basis and Fundamentals. Butterworth-Heinemann, 2005.

30. Ypma T. Historical development of the Newton-Raphson method. SIAM Review 1995; 37(4):531-551.

31. Allen MP, Tildesley DJ. Computer Simulation of Liquids (Reprint edn.) Oxford Science Publications, Oxford University Press: USA, 1989.

32. Desrues J, Viggiani G. Strain localization in sand: an overview of the experimental results obtained in Grenoble using stereophotogrammetry. International Journal for Numerical and Analytical Methods in Geomechanics 2004; 28(4):279-321.

33. Vardoulakis I. Shear band inclination and shear modulus of sand in biaxial tests. International Journal of Numerical and Analytical Methods in Geomechanics 1980; 4(2):103-119.

34. Saint-Cyr B, Szarf K, Voivret C, Azéma E, Richefeu V, Delenne JY, Combe G, Nouguier-Lehon C, Villard P, Sornay P, Chaze M, Radjai F. Particle shape dependence in 2D granular media. Europhysics Letters - EPL 2012; 98(4):44008.

35. Besuelle P, Chambon R, Collin F. Switching deformation modes in post-localization solutions with a quasibrittle material. Journal of Mechanics of Materials and Structures 2006; 3:1115-1134. 